\title{
Forgetting rates in neuropsychiatric disorders
}

\author{
Philip Lewis, Michael D Kopelman
}

\begin{abstract}
Objective-Previous studies have attributed accelerated forgetting rates on recognition memory tasks to temporal lobe pathology, but findings in some patient groups may have been attributable to metabolic disruption. Findings in psychiatric disorders such as schizophrenia are conflicting. The purpose of the present study was to compare forgetting rates in patients with confusional states (postelecroconvulsive therapy (post-ECT), delirium), with those obtained in schizophrenic patients (with putative temporal lobe pathology), non-ECT depressed patients, and healthy controls. The findings could also be compared with previous reports in patients with head injury, focal structural lesions, and
\end{abstract} Alzheimer's dementia.

Methods-Two studies employed a picture recognition task to examine forgetting rates, the first between delays of 1 minute, 15 minutes, and 30 minutes, and the second between delays of 10 minutes, 2 hours, and 24 hours.

Results-There were no significant differences in forgetting rates between 1 minute and 30 minutes, but the ECT group showed accelerated forgetting between 10 minutes and 2 hours compared with healthy controls, associated with a rapid decline in "hit rate". This was not attributable to differential changes in either depression or severity of memory impairment. There were no differences in forgetting rates across the other subject groups. Conclusion-Post-ECT confusional state patients (similarly to "within posttraumatic amnesia" patients with head injury) show accelerated forgetting on a recognition memory task and, in this, they contrast with patients who have focal structural lesions or widespread cortical atrophy. Accelerated forgetting may reflect the effect of disrupted cerebral metabolism on either "consolidation" or memory "binding" processes.

(F Neurol Neurosurg Psychiatry 1998;65:890-898)

Keywords: forgetting; memory; electroconvulsive therapy; schizophrenia

Dr M D Kopelman,

Academic Unit of Psychiatry,

United Medical and Dental

Schools of Guy's and St

Thomas's Hospital, St

Thomas's Campus, Lambeth

Palace Road, London SE1

7EH, UK

Received 6 October 1997

and in final form

28 May 1998

Accepted 15 June 1998 was based originally on findings in one patient, $\mathrm{HM},{ }^{2}$ in whom the results were not replicated when he was tested on different occasions. ${ }^{4}$ Moreover, when the original test procedure ${ }^{12}$ was used in various groups of patients with actual or presumed temporal lobe pathologyincluding patients with Alzheimer's disease, ${ }^{56}$ patients with head injury (out of the period of post-traumatic amnesia), ${ }^{3}$ and patients with anoxic brain damage ${ }^{7}$ - the findings failed to substantiate the original hypothesis. In general, patients with temporal lobe lesions do not forget faster than either healthy controls or other groups of amnesic patients, once learning has been accomplished.

Nevertheless, there are three studies which have shown faster forgetting in patient groups, using this procedure. Squire ${ }^{8}$ showed faster forgetting on a d prime measure in electoconvulsive shock therapy (ECT) patients, who were shown a series of pictures from magazines 2 hours after their electric shock. Secondly, Levin $e t a \beta^{3}$ found faster forgetting in a group of patients with head injury who were still experiencing post-traumatic amnesia (PTA), whereas patients with head injury "out of PTA" forgot normally. Both these groups of researchers attributed the faster forgetting to presumed temporal lobe dysfunction. However, Levin et $a l^{3}$ published CT findings on their individual patients, and close inspection shows that some patients with unequivocal temporal lobe lesions forgot at a normal rate, whereas other patients with lesions outside the temporal lobes manifested faster forgetting than the rest of the group. Thirdly, in a study of normal aging, Huppert and Kopelman ${ }^{9}$ found significant differences in forgetting rates, although they accounted for a relatively small proportion of the total variance in test scores. These authors postulated that glial degeneration in normal aging produces a decline in the metabolic processes necessary for memory retention, but, when this is superceded by dementia, severe loss of neurons (including cholinergic neurons) superimposes a profound acquisition deficit without any further accentuation of the forgetting rate, ${ }^{10}$ relative to age matched nondementing controls. ${ }^{5611}$ Briefly, it seems plausible that the gross metabolic disruption which occurs after $\mathrm{ECT}^{12}$ or in the immediate aftermath of a head injury (the period "within PTA") could be the basis for the faster forgetting found in the studies of Squire ${ }^{8}$ and Levin et $a l,^{3}$ rather than damage to any specific brain structure, such as the temporal lobes. In subtler form, metabolic disturbance might also be the basis for the finding of a moderate degree of accelerated forgetting in normal aging. ${ }^{9}$

To investigate this hypothesis further, there are two requirements: (1) to replicate the finding 
of Squire ${ }^{8}$ in ECT and the result of Levin et al for patients "within PTA", ${ }^{10}$ as nobody seems to have attempted to do so previously; and (2) to carry out a similar study in another group of patients in confusional state or delirium, in whom disrupted metabolic processes could be inferred. The present report describes such a study in (1) post-ECT patients and (2) a mixed group of delirium patients. There were two further components to the study. Firstly, in addition to the conventional Huppert-Piercy procedure, ${ }^{12}$ we also investigated performance on a related task, in which we matched learning or acquisition levels at a 1 minute delay (instead of the 10 minute delay used in the conventional procedure). Forgetting was examined at 15 and 30 minute intervals, instead of delays between 24 hours and 1 week, conventionally used. This was done because various studies (reviewed elsewhere ${ }^{13}$ ) have suggested that differences in forgetting rates might be especially likely to arise within these shorter intervals, although the interpretation of many of these studies has been confounded by "ceiling" and "floor" effects. Secondly, we also included a group of schizophrenic patients in our investigation. The reason for their inclusion was that there is postmortem and neuroimaging evidence of temporal lobe atrophy in schizophrenic patients, ${ }^{14}{ }^{15}$ as well as histological evidence of a reduction or disorganisation of neurons in the CA1 region of the hippocampus and in the entorhinal cortex. ${ }^{16-18}$ Previous investigations of forgetting rates in schizophrenic patients have employed different procedures, and the findings have been conflicting. ${ }^{19}{ }^{20}$ Unlike Shoqeirat and Mayes, ${ }^{19}$ we chose to investigate chronic, rather than acute, schizophrenic patients as we expected that they would be more likely to have temporal lobe pathology. ${ }^{21}$ (We acknowledge that there may also be changes in the frontal lobes or basal ganglia in schizophrenic patients, but it is the temporal lobe abnormalities which have been considered to be most pertinent to forgetting rates. ${ }^{2} 381920$ )

Consequently, the present study investigated (1) ECT treated depressed patients; (2) non-ECT treated depressed patients to examine the effect of depression in the absence of ECT; (3) confusional state (delirium) patients (with presumed metabolic dysfunction); (4) chronic schizophrenic patients (with presumed temporal lobe pathology); and (5) healthy control subjects. It was hypothesised that ECT and confusional state patients, with gross metabolic disruption, would show accelerated forgetting, but that chronic schizophrenic patients with presumed temporal lobe pathology would not show faster forgetting, relative to (non-ECT) depressed patients and healthy controls.

\section{Subjects and methods}

The patients were recruited from the medical and psychiatric wards from Guy's, St Thomas's, South Western, and Bexley Hospitals. In the schizophrenic, ECT, and depressed groups, patients with a history of suspected focal brain pathology, alcoholism or other substance misuse, convulsions, a (previous) course of ECT within the past year, evidence of organic dementia, or a recent change in psychiatric diagnosis were excluded. Patients were diagnosed according to DSM III-R criteria, ${ }^{22}$ except that we allowed some leniency in terms of disorganised thinking in the delirium and confusional state group: criterion B for delirium requires that patients should have "rambling, irrelevant, or incoherent speech". Patients who satisfied criterion B would not have been able to give informed consent to the experiment and certainly would not have been testable.

There were 15 healthy control subjects used in the study, 16 non-ECT depressed patients, 14 patients with chronic schizophrenia, 13 ECT treated depressed patients, and eight patients with delirium (acute confusional state). The ECT patients were tested 2 hours after receiving an electric shock, as Squire ${ }^{8}$ had done. The eight delirium patients included five with extracranial causes: two with renal failure, one with hypercalcaemia, one with probable porphyria, and one with pernicious anaemia giving rise to acute hypoxia. After treatment of his anaemia, this patient did not show any evidence of residual (hypoxic) brain damage. There was one patient whose confusional state remained of uncertain aetiology, but the patient showed pronounced slow waves on EEG. Finally, there were two patients with intracranial causes to their confusion: one patient who had had a head injury and was still within the period of PTA, and one patient who had raised intracranial pressure.

A full psychiatric and medical history was obtained, including a review of the case notes, to establish whether patients could satisfy the criteria for inclusion in the study. A reading test $\left(\right.$ NART-R) ${ }^{23}$ was employed to estimate premorbid intelligence; in schizophrenic patients, the NART has been shown to be valid in those living in the community, ${ }^{24}$ although there may be decrements in institutionalised patients ${ }^{24}$ (11 out of 14 in this group were not living in institutions), and scores in schizophrenic patients seem stable through time. ${ }^{25}$ Six subtests (vocabulary, comprehension, similarities, comprehension, block design, picture completion) from the Wechsler adult intelligence scalerevised (WAIS-R) ${ }^{26}$ were employed to assess current intelligence. The Wechsler memory scale-revised (WMS-R $)^{27}$ was used to determine current anterograde memory performance, using verbal and visual material for both immediate and delayed recall. This scale also gives a measure of current attention and concentration performance. Three different versions of the logical memory story from the original version of the Wechsler memory scale ${ }^{28}$ were also administered to the subjects at intervals (indicated in the appendix) to disclose any changes in memory, and their order was counterbalanced across subjects.

Before the start of the experiment, all subjects were administered the Hamilton depression scale. ${ }^{29}$ The Beck depression scale ${ }^{30}$ was administered to all subjects on each occasion of testing, and (in the ECT group) also on the day before ECT. Finally, the schizophrenic 
Table 1 Background characteristics of subject groups means (SD)

\begin{tabular}{llllll}
\hline & Controls & Schizophrenia & $\begin{array}{l}\text { Depressed } \\
\text { (non-ECT) }\end{array}$ & ECT & Delirium \\
\hline No & 15 & 14 & 16 & 13 & 8 \\
Sex ratio & $5 \mathrm{M}: 10 \mathrm{~F}$ & $9 \mathrm{M}: 5 \mathrm{~F}$ & $8 \mathrm{M}: 8 \mathrm{~F}$ & $8 \mathrm{M}: 5 \mathrm{~F}$ & $5 \mathrm{M}: 3 \mathrm{~F}$ \\
Age (y) & $44.2(9.7)$ & $49.7(12.8)$ & $53.9(13.9)$ & $50.1(11.5)$ & $53.8(18.7)$ \\
Premorbid IQ (NART-R) & $108.3(9.4)$ & $95.6(14.7)$ & $102.0(16.3)$ & $98.3(15.2)$ & $91.3(6.9)$ \\
Current (full scale) IQ (WAIS-R) & $101.3(14.8)$ & $85.1(7.8)$ & $95.6(16.1)$ & $91.3(12.1)$ & $79.5(9.1)$ \\
General memory (WMS-R) & $107.6(16.8)$ & $76.2(13.8)$ & $105.9(21.3)$ & $90.2(19.5)$ & $71.6(20.2)$ \\
Delayed memory (WMS-R) & $109.6(16.5)$ & $78.4(11.9)$ & $100.4(19.4)$ & $84.6(19.9)$ & $60.7(12.9)$ \\
IQ-general memory (means) & -6.3 & 8.9 & -10.3 & 1.1 & 7.9 \\
Hamilton depression score & $1.7(2.5)$ & $12.4(4.9)$ & $21.0(7.7)$ & $22.2(8.8)$ & $8.7(4.1)$ \\
Negative symptoms (SANS) & Not done & $40.3(15.5)$ & Not done & Not done & Not done \\
\hline
\end{tabular}

Values are means (SD).

patients were assessed for the presence and severity of negative symptoms by the use of the scale for the assessment of negative symptoms (SANS). ${ }^{31}$ The appendix shows the order in which tasks were administered during the two experiments below.

Table 1 summarises the background characteristics of the subject groups. The patients did not differ significantly in either age or estimated premorbid IQ (NART-R). On the other hand, the groups differed significantly $(F=4.04, \quad \mathrm{p}<0.01)$ in current pro-rated (WAIS-R) full scale IQ, the delirium group performing poorest because of their confusional state, but the schizophrenic and ECT groups also showing relatively low scores. The groups also differed significantly in terms of WMS-R general memory and delayed memory $(F=9.72, \mathrm{p}<0.01$ and $F=10.05, \mathrm{p}<0.001$ respectively), and in terms of the mean discrepancy between IQ and general memory (MQ) scores $(F=6.71, \mathrm{p}<0.001)$ - the delirium and schizophrenic patients showing mean discrepancies of 7.9 and 8.9 points (IQ-MQ) respectively, indicating impaired memory performance. In the case of the schizophrenic patients, this finding is consistent with various other studies showing anterograde memory impairment in such patients. ${ }^{32-34}$ The groups also differed significantly in mean Hamilton depression scores $(F=26.1, \mathrm{p}<0.001)$, the ECT and non-ECT depressed groups being the most severely and comparably depressed. Negative symptoms on the scale for the assessment of negative symptoms (SANS) ${ }^{31}$ were assessed only in the schizophrenic group, mean score 40.3 (SD 15.5). This represents the sum of the subscores (out of 95), and indicates a high prevalence of negative symptoms, consistent with the clinical diagnosis of chronic schizophrenia.

EXPERIMENT 1: FORGETTING OVER SHORTER DELAYS

Purpose

The aim of this investigation was to examine forgetting rates at 15 minutes and 30 minutes on a recognition memory test, having "matched" initial learning at 1 minute as closely as possible by "titrating" initial exposure times. As indicated in the appendix, this task was performed 1 week after that described in experiment 2 , but we present the findings first because they covered shorter delay intervals.
Procedure

A picture recognition test was modelled on the original Huppert-Piercy ${ }^{12}$ procedure, except that the intervals being investigated were much shorter. Subjects were shown 60 coloured slides of landscapes, people, and objects (taken from magazines) from those in the original study. ${ }^{12}$ Throughout the experiment, slides were presented on a Singer Caramate back projector screen, and slide presentation was timed using a preprogrammed cassette tape. Subjects were asked to view each slide and to name one item from each picture presented.

To match scores on recognition memory testing after a 1 minute delay, exposure times were manipulated such that the mean exposure times were longer for confusional state (delirium) patients than for ECT, depressed, and schizophrenic patients, and longer for each of these groups than for healthy controls. Exposure times for each subject group were initially determined in pilot studies to give initial 1 minute recognition memory (total correct) performance within the range $70 \%$ to $90 \%$ correct, but occasional adjustments to the exposure time for some groups were made to try to match each group's mean recognition performance at 1 minute as closely as possible to those of the other groups. Mean recognition scores at 1 minute in each group were monitored as the study progressed, and occasional alterations were made to the exposure time for subsequent subjects in a given group. The exposure time was made longer if the group mean to date was falling at or below the $70 \%$ mark, and shorter if it was at or above $90 \%$. The mean exposure times per slide in each group at completion of the study were: controls 0.6 seconds (SD 0.2), depression 1.0 seconds (SD 0.0), schizophrenia 1.0 seconds (SD 0.0), ECT 3.4 seconds (SD 1.7), delirium 6.0 seconds (SD 3.1).

In addition, an interstimulus task was carried out between presentation of the individual picture stimuli to "match" the total duration over which the stimuli were presented (and the mean item to test delay ${ }^{35}$ ), taking account of the varying exposure times for the actual target stimuli. In this interstimulus task, subjects were shown slides consisting of a shape (either a black square or a black triangle) and underneath a number of coloured squares or triangles. Subjects were asked to count how many items of the indicated shape there were in the slide. The time they had to perform this 

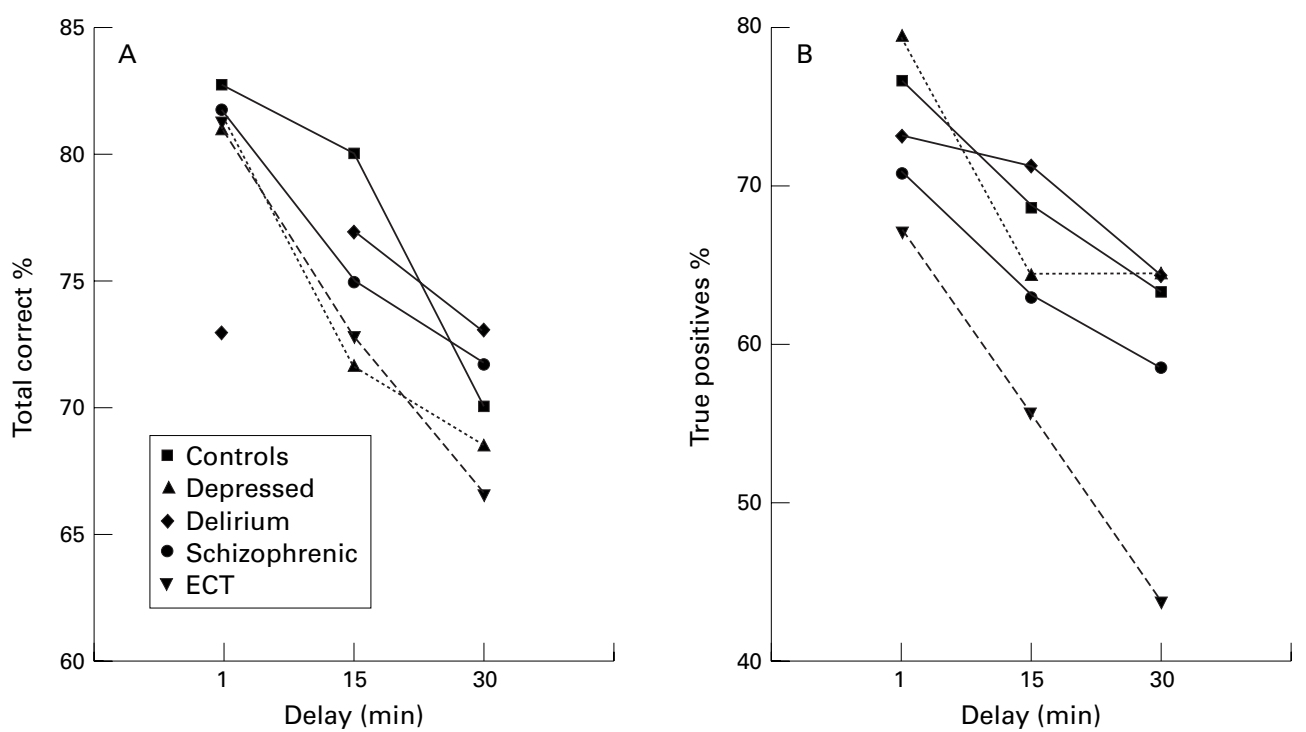

Figure 1 (A) Correct recognition scores (\%), indicating forgetting rates between 1 minute and 30 minutes in experiment 1. (B) Changes in hit rates (true positives) between 1 minute and 30 minute delays in experiment 1.

task was inversely proportional to the time in which the picture stimuli were presented. Subjects continued to perform this interstimulus task after the presentation of the final target stimulus item for 50 seconds until the instructions for the first recognition memory test were given.

Recognition memory testing was performed at 1 minute, 15 minutes, and 30 minutes after presentation of the stimuli. At each delay, 40 stimuli were presented, consisting of 20 targets and 20 distractors: subjects were asked to say whether they had seen each picture before or not (yes/no recognition). Three sets of test material were counterbalanced for each of the three delay intervals.

It should be acknowledged that the Huppert-Piercy procedure of "titrating" exposure times across different subject groups has been criticised in the literature ${ }^{3536}$ because there may be considerable variability in subjects' encoding and attentional processes during the titration period. To measure forgetting rates, the main alternative strategies are: (1) learning-to-criterion, which is a variant, subject to the same criticisms; (2) "matching" by measuring forgetting from varying delay intervals across the subject groups (longer delays for the controls than the patients), but this involves comparisons between the groups across different points in their forgetting curves; (3) comparing selected subgroups of the samples "matched" for initial performance, but this introduces its own biases; or (4) no matching, in which case "like" is not being compared with "like", there are problems in determining the appropriate measurement, ${ }^{37} 38$ and there are serious dangers of ceiling and floor effects. ${ }^{13}$ The great advantage of the Huppert-Piercy technique is that it allows measurement of the forgetting rate from a pragmatically determined starting point, avoiding ceiling and floor effects, and that the exposure time seems to influence the initial level of acquisition rather than the subsequent rate of forgetting. ${ }^{9}{ }^{39}$ The last point means that it is the attaining of a matched starting point which is critical, rather than the specific processes which underlie any individual subject's learning. Further discussion of this topic is beyond the scope of this paper.

Results

Figure 1A shows the pattern of results across the five patient groups for forgetting rates between 1 minute and 30 minute delays. The delirium group could not be matched to the other four groups for initial (1 minute) recognition, but, after manipulation of the exposure times, the other four groups were well matched at the 1 minute delay. At 30 minutes, the groups were scoring well above chance, and the rates of forgetting, as indicated by the slopes of the curves, were very similar. It was also true that the delirium group showed a similar rate of forgetting to the other three patient groups between the 15 minute and 30 minute delays, and this group actually scored higher at 15 minutes than at 1 minute. Overall, including all five groups, there was a significant main effect of delay $(F(2,88)=21.9, \mathrm{p}<0.0001)$ indicating that forgetting occurred through time. However, the main effect of group ( $F$ $(1,44)=0.70)$ and the group by delay interaction effect $(F(8,88)=1.71)$ were not significant, the last indicating no difference in overall forgetting rates. After omitting the delirium group from the analysis, the group by delay interaction effect remained non-significant. We also carried out analysis of these data in terms of the d prime statistic, a measure of signal detection, and this gave consistent results. There was a significant effect of delay ( $F$ $(2,88)=23.5, \mathrm{p}<0.0001)$, indicating that forgetting did occur, but there was no significant main effect of group $(F(1,44)=0.22$, NS), nor was there a significant group by delay interaction effect $(F(8,88)=1.88, \mathrm{NS})$, and this also remained true after excluding the delirium group from the analysis.

Figure 1B shows the findings for hit rate (true positives). Again, there was no significant 
group by delay interaction effect ( $F$ $(8,88)=0.72$, NS), but the figure indicates a (non-significant) trend for the ECT group to show a faster decline in hit rate between 15 and 30 minutes, especially compared with the nonECT depressed group. The delirium group performed similarly to the other patient groups at 1 minute in terms of hit rate despite the fact that this group performed badly in terms of total correct or $\mathrm{d}$ prime scores at 1 minute. This is because, in their confusional state, patients with delirium initially gave a very high rate of false positive as well as true positive responses. At 15 minutes and 30 minutes after presentation of the stimuli, they became more discriminating and more conservative in their strategy, resulting in better total correct scores at 15 minutes than at 1 minute, although it is not clear why this change in strategy arose.

\section{Discussion}

The overall finding was that differences in forgetting rates between the subject groups across these delays did not occur. By using the titration procedure, four of the groups were well matched in terms of recognition memory scores at initial testing, and we avoided obtaining a floor effect (chance performance-that is, $50 \%$ correct) at the later delays. On the other hand, the findings in the delirium group remain equivocal, because we were unable to match this group to the other groups' scores at the 1 minute delay. However, when the 15 and 30 minute delays were taken in isolation, the delirium group did not differ significantly from the other four groups in terms of rate of forgetting.

The present finding is consistent with the results obtained on this same test in a parallel study of patients with organic amnesia, resulting from diencephalic, temporal lobe, or frontal lobe lesions, ${ }^{40}$ and a further study which investigated patients with Alzheimer's disease. ${ }^{41}$ In those studies, none of the patient groups differed significantly from healthy controls in forgetting rates over these delay intervals, and, in particular, patients with temporal lobe lesions did not forget faster than patients with diencephalic pathology, and this was also the case when visually presented words and abstract designs were used as stimulus material.

Finally, the trend for the ECT group to show a rapidly declining hit rate between 15 and 30 minutes is relevant to the interpretation of findings in the next experiment.

EXPERIMENT 2: FORGETTING OVER LONGER DELAYS

Purpose

The aim of this investigation was to examine forgetting rates at 2 hour and 24 hour delays in the same patient groups, after matching initial performance as closely as possible at 10 minutes using the conventional HuppertPiercy procedure. ${ }^{12}$

Procedure

Subjects were exposed to 120 coloured slides of pictures from magazines displayed on a back projector and asked to remember them. During the initial exposure to test material, subjects were asked to name an item seen on each slide to ensure that they were paying attention. At 10 minutes, 2 hours, and 24 hours after the end of the exposure of this test material, sets of slides consisting of 40 of the original pictures and 40 new distractor slides (all in random order) were presented to the subjects as a recognition memory test. Subjects were asked to respond by simple yes or no replies as to whether each slide had appeared in the original set or not.

To measure the rate of forgetting, it was necessary that the subject groups were matched as closely as possible for initial (10 minute) recognition scores. The exposure time per slide was varied between the subject groups to achieve this. Initially, guided by findings in the neuropsychological literature and pretest piloting, exposure times of 0.5 seconds per slide for healthy controls, 1.5 seconds per slide for the non-ECT depression patients, 2.0 seconds for the schizophrenic patients, and 6.0 seconds per slide for the ECT and delirium groups, were selected to try to obtain 10 minute recognition memory scores within the range $70 \%$ to $90 \%$ correct. Subsequently, these exposure times were modified in some of the groups, because the mean recognition score at 10 minutes was monitored in each group as the experiment progressed, and occasional adjustments to the exposure time for subsequent subjects were made to match each group's mean performance at 10 minutes as closely as possible. The final mean exposure times were: controls 0.75 seconds (SD 0.2), depression 1.5 seconds (SD 0.0), schizophrenia 2.0 seconds (SD 0.0), delirium 5.9 seconds (SD 1.7), and ECT 6.5 seconds (SD 1.7). Forgetting was then measured by reassessing recognition memory, using different sets of slides, at 2 hours and 24 hours after initial presentation. In the ECT group, initial testing occurred 2 hours after administration of ECT, and the 2 hour delay (about 4 hours after administration of ECT) was selected to measure forgetting during the recovery phase after ECT. For 10 out of 13 subjects in the ECT group, this experiment was carried out after their first administration of an electric shock treatment; in the remaining three subjects, it was carried out at the second ECT administration. We did not employ an interstimulus distractor task in this experiment, as we wished to make the design as close as possible to previous studies using this procedure over similar delays. ${ }^{1-10}$

\section{Results}

Figure 2A shows the results in this experiment in terms of total correct scores (measuring forgetting from 10 minutes and beyond) across the patient groups. There was a significant main effect of delay $(F(2,88)=155.1$, $\mathrm{p}<0.0001)$, indicating that forgetting occurred, no significant main effect of group ( $F$ $(4,61)=0.44, \mathrm{NS})$, indicating that the groups were well matched for overall level of performance, and no significant group by delay interaction effect $(F(8,122)=1.44, \mathrm{NS})$. However, examining the first two data points only 

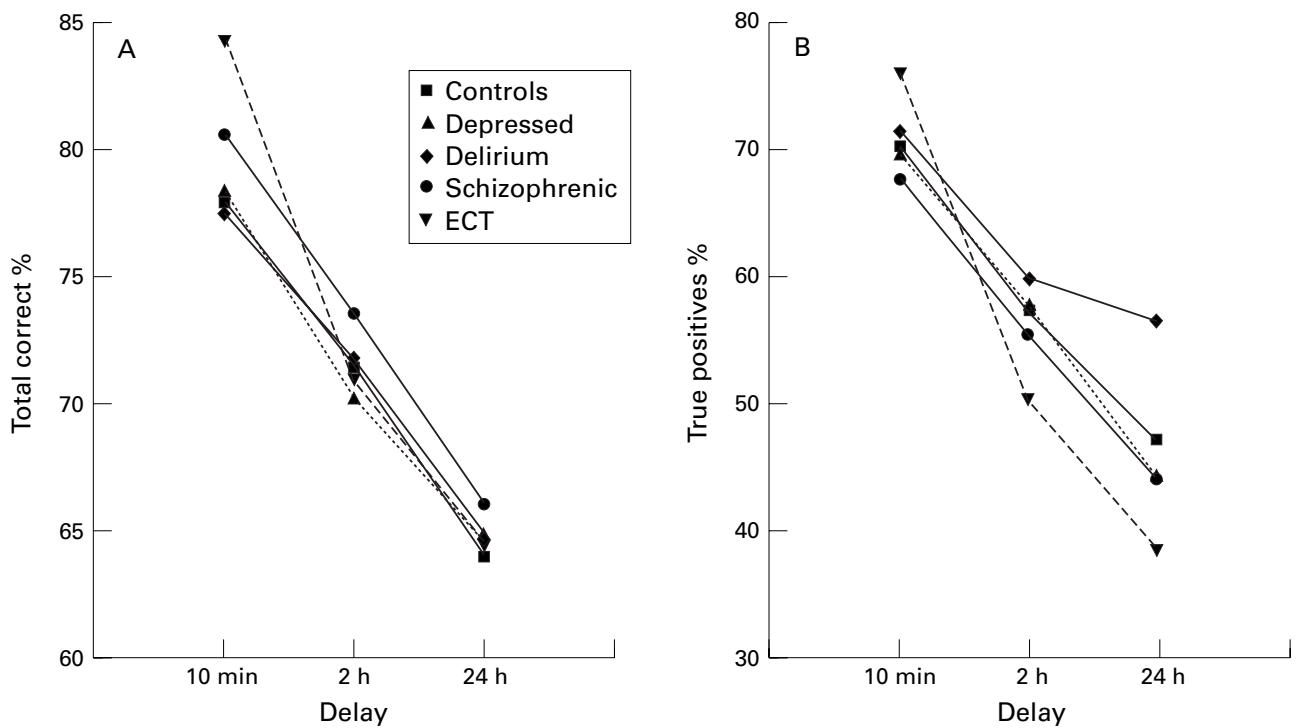

Figure 2 (A) Correct recognition scores (\%), indicating forgetting rates between 10 minutes and 24 hours in experiment 2. (B) Changes in hit rates (true positives) between 10 minute and 24 hour delays in experiment 2.

across all five groups gave a significant group by delay interaction $(F(4,61)=2.56, \mathrm{p}<0.05)$ resulting from faster forgetting in the ECT group between the 10 minute and 2 hour delays. When the ECT group was omitted from this analysis, or when the delays between 2 hours and 24 hours were examined in isolation across all five groups, there were no significant group by delay interaction effects. To test Squire's ${ }^{8}$ hypothesis more specifically (as he had examined fewer groups-namely, ECT patients $v$ themselves some months later, and Korsakoff patients $v$ alcoholic controls), we compared the ECT group with the healthy controls over all three time points. In this case, there was a significant interaction effect ( $F$ $(2,52)=3.36, p<0.05)$, indicating faster forgetting in the ECT group. When the findings were examined in terms of the $d$ prime statistic across all five groups and all three delay intervals, there was again a significant main effect of delay, indicating that forgetting did occur, but the group by delay interaction was not significant $(F(8,122)=0.74, \mathrm{NS})$. However, the slopes of the curves indicated the same trend as before, the ECT group showing a (nonsignificant) trend towards faster forgetting between the 10 minute and 2 hour delays, but no difference from the other four groups between the 2 hour and 24 hour delays.

Figure $2 \mathrm{~B}$ shows the results for hit rate (true positives) only. In this analysis, there emerged a significant group by delay interaction effect $(F$ $(8,122)=2.17, \mathrm{p}<0.05)$, with the ECT patients showing a substantially faster mean forgetting rate than the other four groups between the 10 minute and 2 hour delays. This was confirmed when the ECT group was compared with the healthy controls, taken in isolation (group by delay interaction $F(2,52)=3.58, \mathrm{p}<0.05)$, and also when they were compared with the non-ECT depressed patients $(F(2,54)=4.56$, $\mathrm{p}<0.025)$. There were no significant differences across the other four groups.

To examine whether the faster forgetting rate in the ECT group might simply be attributable to differences in the severity of memory impairment, we examined logical memory scores on three equivalent prose passages. ${ }^{28}$ Immediate recall of one of these prose passages had been tested immediately after each occasion of recognition testing during experiment 2 , the order of administration of the prose passages being counter balanced across subjects. The results are plotted in fig 3A, which clearly indicates that the performance of the ECT group at each delay fell midway between the schizophrenic group and the delirium group, on the one hand, and the healthy controls and non-ECT depressives, on the other. (The differences between the groups on logical memory were significant $(p<0.025)$ on each occasion of testing.) Thus, the faster forgetting obtained in the ECT group cannot be attributed to these subjects having more severely disordered memory, because their logical memory scores were higher than those of the schizophrenic and delirium groups at each occasion of testing.

Similarly, we examined whether differences in forgetting rates could be attributed to changes in the severity of depression. The Beck depression inventory ${ }^{30}$ had been administered to the ECT group on the day before their ECT treatment, and on the first and second day of this experiment as well as a week later (when experiment 1 was conducted). The Beck inventory was also given to the non-ECT depressed group on each day of testing (days 1 and 2 of this experiment and a week later during experiment 1). The schizophrenia, delirium, and control groups completed the Beck inventory on day 1 of this experiment and a week later (see appendix). The findings are shown in fig $3 \mathrm{~B}$, where days 1 and 2 refer to experiment 2 , and day 3 refers to experiment 1 a week later. As expected, there were marked differences in Beck scores across the groups on day $1(F(4,52)=8.80, \mathrm{p}<0.001)$. However, the ECT and depressed group did not differ significantly for mean score on day 1 , nor in terms of the rate of change in score over the 

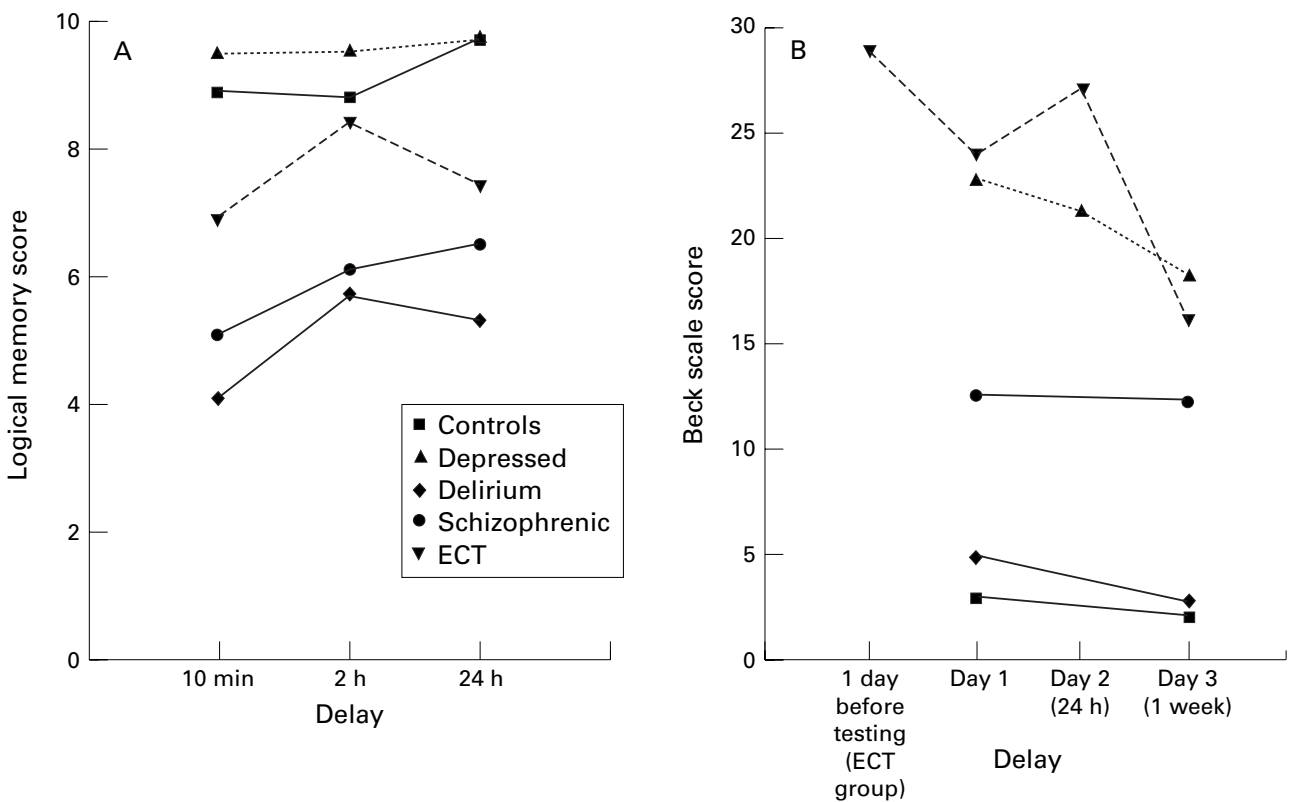

Figure 3 (A) Mean logical memory scores across the subject groups, administered at each delay interval of experiment 2 , using equivalent versions of the logical memory prose passages. (B) Beck depression scores (means for each subject group), assessed at intervals across experiments 1 and 2.

experimental week (group by delay interaction: $F(2,23)=2.67, \mathrm{NS})$. Although there seems to have been a trend for the ECT group to have been more severely depressed than the nonECT depressed group on day 2, this would not have accounted for the differences in forgetting rates which occurred between the 10 minute and 2 hour delays (measured on day 1). It seems very unlikely that the differences in forgetting rates obtained could be accounted for either by differences in the severity of memory impairment or by differential changes in depression across the subject groups.

In terms of a correlational analysis, neither premorbid or current IQ,${ }^{23}{ }^{26}$ general or delayed memory quotients, ${ }^{27}$ nor the depression scores $^{29}{ }^{30}$ correlated significantly with forgetting rates (indicated by initial minus final recognition scores) in either experiments 1 or 2 , although there were significant correlations between current IQ and the memory quotients $(p<0.001)$. In brief, forgetting rates were not related to the general level of anterograde memory (new learning) ability, or to IQ or the severity of depression.

Finally, forgetting rates were unrelated to type or dosage of antidepressant or neuroleptic medication in the depressed and schizophrenic groups in either experiment 1 or 2 .

Discussion

These findings indicate that the ECT group forgot faster than the healthy controls between the 10 minute and 2 hour delays. This was indicated by significant group by delay interaction effects across all five groups between 10 minutes and 2 hours for total correct and hit rate scores, and a trend in the same direction for d prime. (There was also a significant group by delay interaction effect across all the delays when the ECT group was compared with the control group, taken in isolation). This finding is consistent with Squire's ${ }^{8}$ result, in which he obtained accelerated forgetting in an ECT group, relative to performance by an alcoholic Korsakoff group and by his ECT patients' own performance after recovery (4 months later). Our logical memory and Beck depression findings (as well as the correlational analysis) make it very unlikely that the faster forgetting of the ECT group over these intervals could be attributed to changes in the severity of their memory disorder or to differential changes in the severity of their depression. On the other hand, our delirium group, whom we had expected to resemble the ECT group (because of the gross metabolic disruption which follows $\mathrm{ECT}^{12}$ ), performed in fact much more like the other three groups (schizophrenic patients, non-ECT depressed, and healthy controls) on this task.

The principal change in performance of the ECT patients between 10 minutes and 2 hours was that they became more cautious; $42 \%$ of their responses at 10 minutes were "yes" ( $v$ $41.5 \%$ in the non-ECT depressed group), whereas only $31 \%$ were positive at 2 hours ( $v$ $38 \%$ in the non-ECT depressed group). The significant decline in hit rate by the ECT group between 10 minutes and 2 hours in the present experiment is consistent with the nonsignificant trend towards a fall in hit rate by the ECT group between 15 and 30 minutes obtained in experiment 1 (fig 1B).

\section{General discussion}

The findings in experiment 2 confirm Squire's ${ }^{8}$ finding that ECT treated patients show accelerated forgetting of pictorial information at delays beyond 10 minutes. This seemed to occur because of a sharp decline in the hit rate (true positive responses) between the 10 minute and 2 hour delays. This pattern of results was different from that obtained in non-ECT depressed, schizophrenic, or delirium patients, and also from that of healthy 
controls. It also contrasts with the finding of normal forgetting on this task, after appropriate matching of initial performance, typically found in patients with various types of structural lesion giving rise to organic amnesia. ${ }^{14-8}$

On the other hand, the ECT group did not show faster forgetting on a task (experiment 1) measuring forgetting rates between 1 minute, 15 minute, and 30 minute delays. In this respect, the ECT patients resembled recent findings in patients with memory disorders arising from focal temporal lobe, frontal, or diencephalic lesions ${ }^{40}$ or Alzheimer's dementia. ${ }^{41}$ These patient groups also failed to show accelerated forgetting between these delay intervals. However, as noted above, the ECT group did show a non-significant trend towards a faster decline in the hit rate between 15 and 30 minutes (experiment 1), consistent with the overall finding of a significant decline in hit rate and in correct recognition scores between 10 minutes and 2 hours (experiment 2).

A possible explanation for these results might be that they reflect a state-dependent phenomenon: as the ECT patients emerge from their ECT state, they have difficulty remembering information learned soon after ECT. Somewhat against that view is the finding that the ECT patients actually did so well (in terms of hit rate) in the immediate aftermath of the ECT (at the 10 minute delay). Moreover, analysis of the sequential logical memory and Beck depression scores (figs $3 \mathrm{~A}$ and B) makes it unlikely that the accelerated forgetting could result from changes in the severity of the memory impairment or of depression between the "test" episodes.

A second possibility is that ECT has a specific effect on some particular brain structure, which results in accelerated forgetting. Squire $^{8}$ and Levin et $a l^{\beta}$ both suggested that specific dysfunction of the temporal lobes might produce accelerated forgetting. However, many studies now exist which indicate that structural lesions, wherever located, do not in themselves produce faster forgetting, making this explanation most unlikely. ${ }^{14-8} 40$

A third possibility is that the ECT produces faster forgetting by disrupting those metabolic processes essential for retention processes at 10 minutes and beyond-sometimes conceptualised as either physiological "consolidation" or memory "binding". The finding of Levin et al" that patients with head injury "within PTA" show faster forgetting, whereas patients with head injury show normal forgetting once their PTA has ended, could be interpreted as consistent with this argument. On the other hand, against our original hypothesis, we failed to find accelerated forgetting in our delirium group, who performed (in experiment 2) very similarly to the non-ECT depressed and schizophrenic patients as well as the healthy controls. To resolve this issue, it may be necessary in future studies to recruit delirium patients with more specific metabolic abnormalities, and to correlate measures of forgetting rate with PET findings for regional and general cerebral metabolism in various patient groups.

It should also be noted that, although our schizophrenic patients had severely impaired memory according to the WMS-R quotients, they showed a normal forgetting rate on both our measures, despite their abnormal performance on many other memory tasks. ${ }^{32-34}$ Their memory impairment may well be attributable to pathology within the temporal lobes, ${ }^{14-18}$ in which case our findings for the schizophrenic group (anterograde memory impairment without accelerated forgetting on recognition memory measures) are entirely consistent with results obtained elsewhere in patients with temporal lobe lesions. ${ }^{45740}$

In summary, our finding of accelerated forgetting between 10 minutes and 2 hours in ECT patients replicates Squire's ${ }^{8}$ finding of accelerated forgetting at 10 minutes and beyond. This accelerated forgetting did not appear over shorter intervals, nor in other groups of psychiatric patients (non-ECT depressed, schizophrenic, delirium). This pattern of results is similar to that obtained in patients with head injury "within PTA" ${ }^{3}$ but it contrasts with that obtained in patients with structural brain lesions or dementia and, as such, ECT is not a good model for organic amnesia. The explanation for faster forgetting in this group requires further investigation, but it may be attributable to the effect of disrupted cerebral metabolism on either consolidation or binding processes and, thereby, on long term retention.

We thank Dr S Browning for help in recruiting patients, and $\mathrm{Dr}$ F Huppert for kindly supplying the test material. This research was funded by a South East Thames Regional Health Authority research grant.

1 Huppert FA, Piercy M. Dissociation between learning and remembering in organic amnesia. Nature 1978;275:317-8.

2 Huppert FA, Piercy M. Normal and abnormal forgetting in organic amnesia: effect of locus of lesion. Cortex 1979;15: 385-390.

3 Levin, H, High WM, Eisenberg HM. Learning and forgetting during post-traumatic amnesia in head-injured patients. F Neurol Neurosurg Psychiatry 1988;51:14-20.

4 Freed DM, Corkin S, Cohen NJ. Forgetting in HM: a second look. Neuropsychologia 1987;25:461-72

5 Kopelman,MD. Rates of forgetting in Alzheimer-type dementia and Korsakoff's syndrome. Neuropsychologia 1985;23:623-38.

6 Freed DM, Corkin S, Growdon JH, et al. Selective attention in Alzheimer's disease: characterizing cognitive subgroups of patients. Neuropsychologia 1989;27:325-39.

7 McKee RD, Squire LR. Equivalent forgetting rates in longterm memory for diencephalic and medial temporal lobe amnesia. F Neurosci 1992;12:3765-72.

8 Squire LR. Two forms of human amnesia: an analysis of forgetting. F Neurosci 1981;1:635-40.

9 Huppert FA, Kopelman MD. Rates of forgetting in normal ageing: a comparison with dementia. Neuropsychologia 1989;27:849-60.

10 Kopelman MD, Corn TH. Cholinergic blockade as a model for cholinergic depletion: a comparison of the memory deficits with those of Alzheimer-type dementia and the alcoholic Korsakoff syndrome. Brain 1988;111:1079-110.

11 Greene JDW, Baddeley AD, Hodges JR. Analysis of the episodic memory deficit in early Alzheimer's disease: evidence from the doors and people test. Neuropsychologia 1996;34: $537-51$

12 Fink M. Convulsive therapy: theory and practice. New York: Raven Press, 1979

13 Kopelman MD. The new and the old: components of the anterograde and retrograde memory loss in Korsakoff and Alzheimer patients. In: Squire LR, Butters N, eds. The neuropsychology of memory. 2nd ed. New York: Guilford, 1992: 130-46.

14 Brown R, Colter N, Corsellis J, et al. Postmortem evidence of structural brain changes in schizophrenia. Arch Gen Psychiatry $1986 ; 43: 36-42$

15 Crow TJ, Ball J, Bloom S, et al. Schizophrenia as an anomaly of development of cerebral asymmetry. Arch Gen Psychiatry $1989 ; 42: 1031-7$. 
16 Kovelman J, Scheibel A. Performance of schizophrenics on tests sensitive to left or right frontal, temporal or parietal function in neurological patients. Biol Psychiatry 1985;19: 1601-21

17 Jeste D, Lohr J. Hippocampal pathologic findings in schizophrenia. Arch Gen Psychiatry 1989;46:1019-24.

18 Arnold SE, Hyman B, Hoessen G, et al. Some cytoarchitectural abnormalities of the entorhinal cortex in schizophrenia. Arch Gen Psychiatry 1991;48:625-32.

19 Shoqeirat M, Mayes A. Spatiotemporal memory and rate of forgetting in acute schizophrenics. Psychol Med 1988;18 843-53.

20 Calev A, Edelist S, Kugelmass S, et al. Performance of longstay schizophrenics on matched verbal and visuo-spatial recall tasks. Psychol Med 1991;21:655-60.

21 Crow TJ. Molecular pathology of schizophrenia; more than one disease process? BMF 1980;280:66-8.

22 American Psychiatric Association. Diagnostic and statistical manual of mental disorders, 3rd edition, revised. Washington DC: APA, 1987.

23 Nelson HE, Willison FR. The National adult reading test. 2nd ed. Windsor: NFER-Nelson, 1991.

24 Crawford JR, Besson JAO, Bremner M, et al. Estimation of premorbid inteliigence in schizophrenia. $\mathrm{Br} \mathcal{F}$ Psychiatry 1992;161:69-74.

25 Smith D, Roberts S, Brewer W, et al. Test-retest reliability of the national adult reading test (NART) as an estimate of premorbid IQ in patients with schizophrenia. Cognitive Neuropsychiatry 1998;3:71-80.

26 Wechsler D. Wechsler adult intelligence scale — revised. London: Psychological Corporation, 1981.

27 Wechsler D. Wechsler memory scale-revised. London: Psychological Corporation, 1987.

28 Wechsler D. A standard memory scale for clinical use. $7 P_{s y}$ chol 1945;19:87-95.
29 Hamilton M. Development of a rating scale for primary depressive illness. British fournal of Social and Clinical Psychology 1967;6:849-60.

30 Beck AT, Ward CH, Mendelson M, et al. An inventory for measuring depression. Arch Gen Psychiatry 1961;4:561-71.

31 Andreasen NC. The scale for the assessment of negative symptoms (SANS). Iowa City, IA: University of Iowa, 1993.

32 Cutting J. Memory in functional psychosis. 7 Neurol Neurosurg Psychiatry 1979;42:1031-7.

33 Calev A. Recall and recognition in chronic non-demented schizophrenics: use of matched tasks. $\mathcal{F}$ Abnorm Psychol 1984;93:172-7.

34 McKenna P, Tamlyn D, Lund C, et al. Amnesic syndrome in schizophrenia. Psychol Med 1990;20:967-72.

35 Mayes AR. Learning and memory disorders and their assessment. Neuropsychologia 1986;24:25-50.

36 Mayes AR. Human organic memory disorders. Cambridge: Cambridge University Press, 1988.

37 Loftus GR. Observations-evaluating forgetting curves. $\mathcal{f}$ Exp Psychol Learn Mem Cogn 1985;11:397-406.

38 Slamecka NJ. Observations on comparing rates of forgetting: comment on Loftus. 7 Exp Psychol Learn Mem Cogn 1985;11:812-6.

39 Huppert FA, Piercy M. Recognition memory in amnesic patients: effect of temporal context and familiarity of material. Cortex 1976;12:3-20.

40 Kopelman MD, Stanhope N. Rates of forgetting in organic amnesia following temporal lobe, diencephalic, or frontal lobe lesions. Neuropsychology 1997;11:343-56.

41 Christensen H, Kopelman MD, Stanhope N, et al. Rates of forgetting in Alzheimer dementia. Neuropsychologia 1998; 36:547-58.

\section{Appendix}

A: Testing schedule for controls, schizophrenic patients, and delirium and depressive patient

Day 1 :

Sign consent form

Show 1st 120 Huppert-Piercy (H-P) slides

NART-R

Beck depression inventory (Beck)

10 minute recognition memory test for $\mathrm{H}-\mathrm{P}$ slides

Logical memory story

Hamilton depression scale (Hamilton)

WMS-R

WAIS-R (6 subtests)

2 hour recognition memory test for H-P slides

Logical memory story

SANS scale (schizophrenia patients only)

Day 2:

Logical memory story

(Beck for depressed patients)

Day 8:

Show short H-P originals (60 slides) + interstimulus distractor task

1 minute recognition memory test

Beck scale

15 minute and 30 minute recognition memory tests

B: Testing schedule for ECT patients

Day before ECT:

Consent form

NART-R

Beck scale

Hamilton

Day of 1st ECT:

2 hours post- ECT:

Show 1 st $120 \mathrm{H}-\mathrm{P}$ slides

Beck scale

10 minute recognition memory test

Logical memory story

WMS-R

WAIS-R

2 hour recognition memory test

Day after ECT:

Logical memory story

24 hour recognition memory test

Logical memory story

Beck scale

Day 8 (approx):

2 hours post-ECT:

usually 3rd ECT

Show short H-P originals (60 slides) + interstimulus distractor task

1 minute recognition memory test

Beck scale

15 and 30 minute recognition memory tests 